\title{
Prevalence of Salmonella enterica serovar 4,[5],12:i:- in England and Wales, 2010
}

K L Hopkins (katie.hopkins@hpa.org.uk)1, E de Pinnaํㅣ J Wain ${ }^{1}$

1. Health Protection Agency Colindale, London, United Kingdom

Citation style for this article:

Hopkins KL, de Pinna E, Wain J. Prevalence of Salmonella enterica serovar 4,[5],12:i:- in England and Wales, 2010. Euro Surveill. 2012;17(37):pii=20275. Available online: http://www.eurosurveillance.org/ViewArticle.aspx?Articleld=20275

Difficulties in accurately identifying serovar 4,[5],12:i:as monophasic variants of Salmonella enterica serovar Typhimurium mean there is confusion in the reporting of serovars Typhimurium and 4,[5],12:i:-. To gain insight into the prevalence and diversity of these monophasic variants in England and Wales, screening for $f l j B$, hin and the serovar 4,[5],12:i:- DT193-associated genomic island was conducted on 609 S. enterica isolates designated as definitive phage type (DT) 193, and 142 isolates serologically-defined as monophasic variants of serovar Typhimurium but belonging to phage types other than DT193. All latter 142 isolates were subtyped by multilocus variable-number tandem repeat analysis (MLVA). MLVA was also applied to 70 DT193 serologically-defined monophasic variant isolates. Results indicate that serovar 4,[5],12:i:- accounted for 108 of 209 (52\%) of DT193 isolates with available serological data and 99 of $142(70 \%)$ monophasic variant isolates belonging to other phage types. Of 609 DT193 isolates, 463 (76\%) lacked fljB and hin. Moreover, genetically-related isolates of DTs 120, 191, 191a, 195, phage types $U_{311}$ and $U_{323}$, and reacts but does not conform (RDNC) and untypable (UT) strains were also lacking either hin and/or fljB. Of note, the serovar 4,[5],12:i:DT193-associated genomic island was identified in not only 458 of 463 (99\%) monophasic DT193 isolates, but also 25 of 139 (18\%) biphasic DT193 isolates and 56 of 76 (74\%) monophasic variants of other phage types. Accurate monitoring of the emergence of serovar 4,[5],12:i:- isolates is important to ascertain the public health impact of these strains; since 2012 the Health Protection Agency's Salmonella Reference Unit has therefore begun determining full antigenic structures of all presumptive 0:4 isolates in addition to routinely performing phage typing for identification of variants of serovar Typhimurium.

\section{Introduction}

Salmonella enterica serovar 4,[5],12:i:- strains failing to express the fljB-encoded phase-2 flagellar antigen have been increasingly isolated from food animals and humans in the European Union (EU) over the last two decades [1]. Such strains were rarely identified before the mid-1990s but according to Enter-Net data, serovar 4,[5],12:i:- was the fourth most common serovar isolated from humans in the EU in 2006 [2]. Nevertheless, as these strains cannot be fully typed by conventional serotyping, it is likely that serovar 4,[5],12:i:- is underreported, principally due to difficulties in differentiating it from serovar Typhimurium, with which it shares antigenic and genotypic similarities $[3,4]$. According to the Kauffmann-White scheme the two serovars share the same 0 -antigens and phase $1 \mathrm{H}$-antigen, but serovar 4,[5],12:i:- lacks expression of the phase 2 flagellar antigen and is therefore considered a monophasic variant of Typhimurium. Problems with the procedure of flagellar phase inversion, which can be time-consuming and technically demanding, and lack of standardisation on how many times phase inversion should be repeated to be confident that an isolate is monophasic may result in misclassification of these two serovars. This process may also be complicated by serovar Typhimurium strains in which serological detection of the phase-2 flagellar antigen is inconsistent [5].

Most isolates of serovar 4,[5],12:i:- can be designated as a recognised phage type using the phage typing scheme of Anderson et al. [6]. In busy reference laboratories where phage typing is used in lieu of full serotyping to identify strains of serovar Typhimurium this may result in misclassification of monophasic isolates as serovar Typhimurium [7]. Several phage types have been associated with recent emergence of monophasic Typhimurium strains [7]. Serovar 4,[5],12:i:- belonging to phage type $\mathrm{U}_{302}$ and expressing mainly resistance to ampicillin, chloramphenicol, gentamicin, streptomycin, sulfamethoxazole, tetracyclines and trimethoprim (R-type ACGSSUTTm) emerged in humans and pig or pork products in Spain in the late 1990 s to subsequently become the fourth most common serovar identified between 1998 and 2000 [8]. In the last ten years serovar 4,[5],12:i:- definitive phage type (DT) 193, and to a lesser extent DT120, expressing resistance to ampicillin, streptomycin, sulphonamides and tetracycline (R-type ASSuT) have rapidly emerged within several European countries in humans $[7,9,10,11]$. Infections have been linked mainly to pigs and pork products, and occasionally to other food animals. More recently, England and Wales has seen the emergence of serovar 
4,[5],12:i:- belonging to previously undefined phage type DT191a associated with frozen reptile feeder mice imported from the United States [12]. As well as belonging to a new phage type, these isolates were unable to utilise dulcitol and expressed resistance to tetracyclines only (R-type T) as has been previously reported in serovar 4,[5],12:i:- isolates from North and South America $[13,14]$. Recently a novel genomic island was identified in multidrug-resistant serovar 4,[5],12:i:DT193 from several European countries, which is being further investigated with respect to virulence properties and metabolic functions to determine whether its acquisition may have contributed to the rapid emergence of this strain [15].

Salmonella strains submitted by primary diagnostic laboratories to the Health Protection Agency's (HPA) Salmonella Reference Unit (SRU) with a preliminary identification as serovar Typhimurium are only phage typed and not routinely subjected to further serological examination unless the phage typing results are inconclusive. Conversely, isolates of serovar 4,[5],12:i:were not routinely phage typed until July 2010. In order to gain a clearer picture of the prevalence and diversity of serovar 4,[5],12:i:- strains isolated in England and Wales during January-December 2010, all S. enterica isolates designated as phage type DT193, and all isolates that were determined to be monophasic variants of phage types other than DT193 on the basis of the Kauffmann-White scheme were screened by polymerase chain reaction (PCR) for the presence of $f l j B$ (encoding the phase-2 flagellar antigen) and hin (facilitates inversion of a promoter element to regulate flagellar phase inversion). In addition, PCR was used to screen for the presence of the serovar 4,[5],12:i:- DT193associated genomic island [15]. Multilocus variablenumber tandem repeat analysis (MLVA) was applied to all serologically-defined monophasic variant isolates of phage types other than DT193 and a subset of DT193 monophasic variants.

\section{Methods}

\section{Bacterial isolates}

The study panel consisted of all $S$. enterica isolates $(\mathrm{n}=609)$ designated as phage type DT193 submitted to the HPA between January-December 2010 for which antigenic structures were readily available for 209 isolates. Of these, the phase-2 flagellar antigen was serologically-detected in 88 isolates: two of serovar 1,4,12:i:1,2, nine of serovar 1,4,5,12:i:1,2, 15 of $4,12: i: 1,2$ and 62 of $4,5,12: i: 1,2$. The phase-2 flagellar antigen was not serologically detected in the 121 remaining isolates: two were serovar 1,4,5,12:i:-, 11 were 4,12:i:- and 108 were 4,5,12:i:-.

Also included in this study were all isolates $(n=142)$ determined to be serologically monophasic (i.e. exhibiting antigenic structures 1,4,12:i:- $(n=5), 1,4,5,12: \mathrm{i}:-$ $(n=12), \quad 4,12: i:-\quad(n=26)$ or $4,5,12: i:-(n=99))$ but belonging to phage types other than DT193 between January-December 2010.

Isolates were submitted to the HPA SRU and originated from human clinical specimens $(n=624)$, animals $(n=40)$, food products $(n=26)$, animal feed $(n=8)$, environmental isolates $(n=3)$ and an unknown source $(n=50)$.

\section{Strain characterisation}

Serology was performed according to the KauffmannWhite scheme and phage typing performed in accordance with HPA protocols $[6,16]$. Isolates that did not react with any of the typing phages were screened using a PCR targeting the malic acid dehydrogenase ( $\mathrm{mdh}$ ) gene of serovar Typhimurium [17]. PCRs targeting $f l j B$ and hin were performed as previously described [5].

\section{Susceptibility testing}

Susceptibility to a panel of 18 antimicrobials was determined by a breakpoint method in Isosensitest agar (Oxoid, Basingstoke, UK). The final plate concentrations $(\mu \mathrm{g} / \mathrm{mL})$ used routinely by the HPA on the basis of long-term studies were: ampicillin (A; 8), chloramphenicol $(C ; 8)$, gentamicin $(G ; 4)$, kanamycin $(K ; 16)$, neomycin $(\mathrm{Ne} ; 8)$, streptomycin $(S ; 16)$, sulphonamides (Su; 64), tetracycline $(T ; 8)$, trimethoprim $(\mathrm{Tm} ; 2)$, furazolidone (Fu; 8), nalidixic acid ( $\mathrm{Nx} ; 16)$, ciprofloxacin ((low-level (Cpl); 0.125); (high-level (Cp); 1)), amikacin (Ak; 4), cephalexin $(C x ; 16)$, cephradine $(C r ; 16)$, cefuroxime (Cf; 16), ceftriaxone $(C n ; 1)$ and cefotaxime $(C t ; 1)$.

\section{Detection of serovar 4,[5],12:i:- DT193-}

associated genomic island

Multiplex PCR to discriminate between free, prophagecontaining and island-carrying forms of the thrW tRNA locus was performed using previously described primers [15].

\section{Multilocus variable-number tandem repeat analysis subtyping}

A total of 212 isolates defined as serologically monophasic were subjected to MLVA according to a previously described protocol [18]. This comprised the 142 monophasic variant isolates of phage types other than DT193 (of which 99 (70\%) were serovar 4,[5],12:i:-) and 70 randomly selected monophasic variants of DT193 (of which 63 (90\%) were serovar 4,[5],12:i:-). Multilocus variable-number tandem repeat analysis profiles were assigned based on the fragment size amplified from each locus, with 'NA' used to denote a locus not present [19]. Standard minimum spanning trees generated in the Bionumerics software package (version 6.1; Applied Maths, Sint-Martens-Latem, Belgium) using the single and double locus variance priority rules were used to visualise the relationships between isolates. Clonal complexes were created based on maximum neighbour distance of changes at one locus and a minimum of five MLVA profiles per complex. 


\section{Results}

\section{Phage typing}

The serologically-determined monophasic isolates $(n=142)$ belonged to phage types DT120 $(n=44$; of which 29 (66\%) were serovar 4,[5]12:i:-), DT191a $(n=33$; of which 31 (94\%) were serovar 4,[5]12:i:-), U323 ( $n=12$; of which all were serovar 4,[5]12:i:-), reacts but does not conform (RDNC) $(n=9$; of which $4(44 \%)$ were serovar 4,[5]12:i:-), U311 ( $n=7$; of which $3(43 \%)$ were sero$\operatorname{var} 4,[5] 12: \mathrm{i}:-)$ and between one to five isolates each of DT7, DT97, DT135, DT191, DT195, DT208, U302 (of which 6 of 16 (38\%) were serovar 4,[5],12:i:-). A further 21 isolates were untypable (UT); all of which were positive for mdh, therefore variants of serovar Typhimurium (of which 14 (67\%) were serovar 4,[5],12:i:-).

\section{Detection of $f l j B$ and hin by}

\section{polymerase chain reaction}

Overall, 463 (76\%) of 609 DT193 isolates were PCRnegative for both fljB and hin (fljB-/hin-). A further four isolates were positive for fljB only ( $f l j B+/ h i n-)$ and three isolates were positive for hin only (fljB-/hin+). Serological detection of the phase-2 flagellar antigen largely agreed with detection of fljB by PCR, with only eight of 209 isolates where the phase-2 flagellar antigen was reportedly detected in isolates that were $\mathrm{fljB}$-/ hin-. Conversely, there were three isolates where the phase-2 flagellar antigen was not detected in isolates that were positive for both $f l j B$ and $h i n(f l j B+/ h i n+)$.

Seventy-six (54\%) of the 142 monophasic isolates of other phage types were fljB-/hin-. Of these, 33 were DT120, 18 were UT, 12 were $U_{323}$, six were $U_{311}$, five were DT195 and one isolate each of DT97 and U302. A further 35 isolates were fljB-/hin+, of which 28 were DT191a, four isolates of RDNC and three isolates of DT191, and seven isolates were $f l j B+/$ hin-, of which six were DT120. The twenty-four isolates that were $f l j B+1$ hin + belonged to a variety of phage types (DT7, DT120, DT135, DT191, DT191a, DT208, RDNC and UT).

\section{Source of monophasic ( $\mathrm{fljB}$-/hin-) isolates}

Three hundred and ninety-nine of the 463 (86\%) DT193 fljB-/hin-isolates were from humans, with the remainder isolated from animal feed $(n=5)$, a cat $(n=1)$, cattle $(n=7)$, lamb meat $(n=1)$, pigs $(n=12)$, pork meat products $(n=12)$ and an unknown source $(n=26)$. The travel history section was completed for only 153 of the 399 (38\%) submission forms. A recent history of travel abroad was reported for 43 of $153(28 \%)$ patients, with travel to the African continent, Asian continent, Cambodia, Egypt, Greece, Hungary, Iraq, Italy, Japan, Mexico, Norway, Qatar and Turkey (each 1 patient), France and Vietnam (each 3 patients), Spain (5 patients) and Thailand (17 patients); the remaining 110 patients did not travel abroad prior to acquiring Salmonella.

Fifty-seven of $76(75 \%)$ fljB-/hin- monophasic isolates of other phage types were from humans, with the remainder isolated from cattle $(n=1)$, pigs $(n=5)$, pork meat products $(n=6)$ and an unknown source $(n=7)$. A travel history was provided for only 15 of 76 patients, with recent travel to Malta, Portugal and Spain (each 1 patient) reported; the remaining 12 patients did not travel abroad prior to acquiring Salmonella.

\section{Susceptibility testing}

Among the 609 DT193 isolates, 364 (60\%) expressed resistance to ampicillin, streptomycin, sulphonamides and tetracyclines (R-type ASSuT) only; among $463 \mathrm{fljB}$-/ hin- DT193 isolates 332 (72\%) were R-type ASSuT. Other common resistance profiles among fliB-/hin- DT193 isolates were resistance to ampicillin, streptomycin and sulphonamides (R-type ASSu) in $50(11 \%)$ isolates and resistance to tetracyclines only (R-type T) in 19 (4\%) isolates. Eleven isolates ( $2 \%$ ) were fully sensitive to all antimicrobials in the test panel.

Among the 142 monophasic isolates of other phage types the most common resistance profiles were R-type ASSuT (15 isolates of DT120, 11 untypable (UT) isolates, six isolates of $U_{311}$, four isolates of $U_{32}$ and one each of DT7, DT195 and U302) and resistance to tetracyclines only (25 isolates of DT191a, four of DT120, three each of DT191 and U323, two of RDNC and one UT). Twentyeight isolates $(20 \%)$ were fully sensitive to all antimicrobials in the test panel, of which 13 isolates were DT120, five were DT191a, four were RDNC, two each of DT191 and DT208, and one each of DT135 and U323.

\section{Occupancy of the thrW site}

Among the $463 \mathrm{fljB}$-/hin- DT193 isolates, 284 (61\%) produced two respective amplicons of 1,128 base pairs (bp) and $903 \mathrm{bp}$ spanning the thrW site between genes proA and STM0325 and indicative of presence of the serovar 4,[5],12:i:- DT193-associated genomic island (Table 1). A further $174(38 \%)$ produced a single amplicon of $903 \mathrm{bp}$. The island was also present in nine $\mathrm{flj} B+/ \mathrm{hin}+$ isolates, and a further $16 \mathrm{fljB}+/ \mathrm{hin}+$ and one $\mathrm{fljB}-/ \mathrm{hin}+$ isolate produced a single $903 \mathrm{bp}$ amplicon.

Thirty-eight $(50 \%)$ of $\mathrm{fljB}$-/hin- isolates of other phage types than DT193 produced amplicons indicative of presence of the island (Table 1); these isolates belonged to DT120, UT, U323 and DT195. A further 18 (24\%) fljB-/hin- isolates belonging to DT120 and U 323 produced a $903 \mathrm{bp}$ amplicon only. The island was also present in five DT12o fljB+/hin-, two fljB+/hin+ isolates belonging to DT120 and RDNC, and one DT120 fljB+/ hin+ isolate produced a single $903 \mathrm{bp}$ amplicon.

\section{Multilocus variable-number tandem repeat analysis subtyping}

A total of 212 isolates, which consisted of 70 randomly selected DT193 serologically-defined monophasic isolates and all serologically-defined monophasic isolates of other phage types were subjected to MLVA subtyping. Fragment analysis identified 51 different profiles, within which two clonal complexes could be identified: clonal complex-1 (CC1) consisting of 29 profiles (155 
Occupancy of the thrW site in Salmonella enterica serovar Typhimurium DT193 and monophasic variants belonging to DT193 and other phage types, England and Wales, 2010 ( $n=751$ isolates)

\begin{tabular}{|c|c|c|c|c|c|c|}
\hline \multirow[t]{2}{*}{$\begin{array}{l}\text { Results of PCRs targeting } \\
\mathrm{fljB} / \mathrm{hin}\end{array}$} & \multirow[t]{2}{*}{ Phage type } & \multicolumn{4}{|c|}{$\begin{array}{c}\text { Results of multiplex PCR targeting the thrW site } \\
\text { Amplicon sizes (in base pairs) }{ }^{\mathrm{a}}\end{array}$} & \multirow[t]{2}{*}{ Number of isolates } \\
\hline & & 1,128 & 903 & 663 & 432 & \\
\hline \multirow[t]{5}{*}{ fljB-/hin- } & \multirow[t]{5}{*}{ DT193 } & + & + & - & - & 284 \\
\hline & & - & + & - & - & 174 \\
\hline & & - & - & + & - & 3 \\
\hline & & - & - & - & + & 1 \\
\hline & & - & - & - & - & 1 \\
\hline \multirow[t]{2}{*}{ fljB-/hin+ } & \multirow[t]{2}{*}{ DT193 } & - & + & - & - & 1 \\
\hline & & - & - & - & + & 2 \\
\hline fljB+/hin- & DT193 & - & - & + & - & 4 \\
\hline \multirow[t]{5}{*}{ fljB+/hin+ } & \multirow[t]{5}{*}{ DT193 } & + & + & - & - & 9 \\
\hline & & - & + & - & - & 16 \\
\hline & & - & - & + & - & 96 \\
\hline & & - & - & - & + & 12 \\
\hline & & - & - & - & - & 6 \\
\hline \multirow{5}{*}{ fljB-/hin- } & \multirow[t]{5}{*}{ Other than DT193 } & + & + & - & - & 38 \\
\hline & & - & + & - & - & 18 \\
\hline & & - & - & + & - & 12 \\
\hline & & - & - & - & + & 2 \\
\hline & & - & - & - & - & 6 \\
\hline fljB-/hin+ & Other than DT193 & - & - & - & + & 35 \\
\hline \multirow[t]{3}{*}{ fljB+/hin- } & \multirow[t]{3}{*}{ Other than DT193 } & + & + & - & - & 5 \\
\hline & & - & - & + & - & 1 \\
\hline & & - & - & - & + & 1 \\
\hline \multirow[t]{5}{*}{ fljB+/hin+ } & \multirow[t]{5}{*}{ Other than DT193 } & + & + & - & - & 2 \\
\hline & & - & + & - & - & 1 \\
\hline & & - & - & + & - & 4 \\
\hline & & - & - & - & + & 16 \\
\hline & & - & - & - & - & 1 \\
\hline
\end{tabular}

DT: definitive phage type; PCR: polymerase chain reaction.

A ' + ' indicates 'presence' while a '-" indicates 'absence' of an amplicon of given size.

according to Trüpschuch et al. [15] amplicons of the multiplex PCR indicate three different occupancy possibilities: amplicons of 1,128bp and $903 \mathrm{bp}$ for presence of the genomic island, $663 \mathrm{bp}$ for prophage-occupied site and $432 \mathrm{bp}$ for the free locus.

isolates) and clonal complex-2 (CC2) consisting of nine profiles (41 isolates) (Figure).

Ten MLVA profiles were shared by six or more isolates accounting for $66 \%$ of isolates (Table 2). All but one of these MLVA profiles was shared by more than one phage type (Figure, Table 2). Forty-three (98\%) of 44 DT120 isolates and 67 (96\%) of 70 DT193 isolates were located in $\mathrm{CC}_{1}$, whilst 29 (94\%) of 31 DT191a isolates were located in $\mathrm{CC}_{2}$. All $\mathrm{CC}_{1}$ isolates failed to amplify the Typhimurium-specific virulence plasmid pSLTbound locus STTR10, whereas all isolates in $\mathrm{CC}_{2}$ produced an amplicon.

Clonal complex 1 and $\mathrm{CC}_{2}$ also correlated well with fljB/hin PCR results and occupancy of the thrW site.
One hundred and thirty-nine (90\%) of $\mathrm{CC}_{1}$ isolates were $\mathrm{fljB}$-/hin- and 34 ( $83 \%$ ) of $\mathrm{CC}_{2}$ isolates were $\mathrm{fljB}$-/ hin+, although identical MLVA profiles were shared by fljB-/hin-, fljB+/hin+ and fljB+/hin- isolates in $\mathrm{CC}_{1}$, and by fljB-/hin+ and fljB+/hin+ isolates in $\mathrm{CC}_{2}$. All but one isolate harbouring the serovar 4,[5],12:i:- DT193associated genomic island were located in $\mathrm{CC}_{1}$, regardless of phage type or fljB/hin PCR results.

\section{Discussion}

Serotyping using the widely accepted KauffmannWhite scheme is central to the epidemiological classification of Salmonella strains and thus to surveillance studies, to identify trends in disease transmission, and for detection of outbreaks. Nevertheless, in recent years there has been a move towards development of 
DNA-based techniques to replace or augment serological testing, and such methods may be used as a basis to define strains as serovar 4,[5],12:i:- or Typhimurium $[20,21,22]$. The range of mechanisms that can result in non-expression of the phase-2 flagellar antigen may mean that development of a reliable molecular serotyping scheme is complex. Lack of phase-2 flagellar antigen expression may be due to different mutations and deletions in $f l j B$, fljA (encoding a repressor of the phase-1 flagellin gene fliC) and hin [5,23,24]. Alternatively, the invertible promoter that controls expression of $f l j B$ and fliC may become locked in one position, thereby allowing only expression of the flic-encoded phase-1 flagellum [23]. This range of mechanisms that contribute to lack of $f l j B$ expression means that there can be discrepancies in detection of the phase-2 flagellum between conventional and molecular serology. The data presented here suggest that conventional serology is adequate for detection of serovar 4,[5],12:i:- DT193, as serological detection of the phase-2 flagellar antigen agreed with detection of $f l j B$ by PCR in $95 \%$ of isolates. Discrepancies between conventional and molecular serology were more apparent in monophasic isolates of other phage types, where $22 \%$ of monophasic isolates were $f l j B+$ by PCR. This may become problematic if any of these strains persist and amplify to the same extent as serovar 4,[5],12:i:- DT193. Identification of not only fljB-/hin- monophasic isolates, but also $\mathrm{fljB}+/$ hin- and fljB-/hin + isolates supports previous suggestions that serovar 4,[5],12:i:- represents multiple genotypes that have emerged independently from serovar Typhimurium $[5,24]$.

Numbers of DT193 isolated in England and Wales have increased from $5.3 \%$ of all serovar Typhimurium in 2000 to $28 \%$ in 2010 , with the number of DT193 R-type ASSuT increasing from $28 \%$ to $62 \%$ over the same time period (HPA Salmonella database, unpublished data). In this study 399 of 509 (78\%) human DT193 isolates were found to be fljB-/hin- monophasic variants, thereby providing strong evidence that the emergence of serovar 4,[5],12:i:- DT193 is contributing to the increase in DT193 isolates from cases of human infection in England and Wales as previously suggested [7]. In addition, we identified multiple isolates of phage types U311, U323 and DT195. Monophasic isolates of serovar 4,[5],12:i:- U311 have previously been reported in Italy, France and Spain $[7,25]$. This is, however, the first time we have identified serovar 4,[5],12:i:- belonging to phage types $\mathrm{U}_{323}$ and DT195, thereby adding to the diversity of phage types already associated with this serovar [7].

Recently, an $18.4 \mathrm{~kb}$ novel genomic island was identified in $90 \%$ of serovar 4,[5],12:i:- DT193 isolated from humans between 2001 and 2008 in Germany and in similar isolates from Luxembourg, Austria, France, Italy and the Netherlands, therefore was proposed as an additional epidemiological marker for the serovar 4,[5],12:i:- DT193 clone emerging across Europe [15]. In this study only $61 \%$ of $f l j B-/$ hin- DT193 isolates produced two amplicons representative of carriage of
FIGURE

Minimum spanning tree of multilocus variable-number tandem repeat analysis of monophasic Salmonella Typhimurium isolates, England and Wales, 2010 (n=212 isolates)

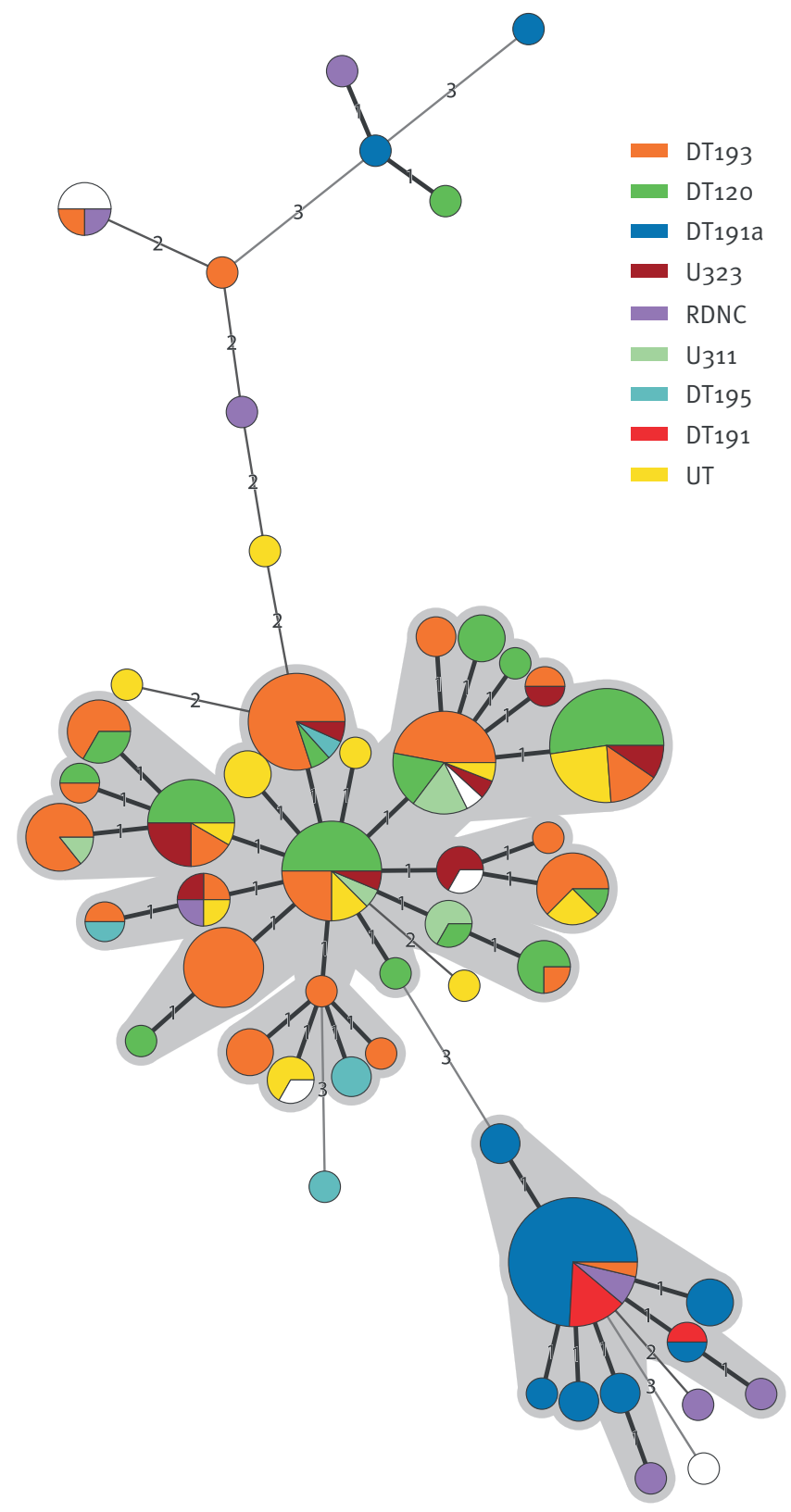

DT: definitive phage type; MLVA: multilocus variable-number tandem repeat analysis; RDNC: reacts but does not conform; UT: untypable.

Node size is proportional to the number of strains with that MLVA profile. Wedges within nodes represent the proportion of isolates with that MLVA profile that belong to a specific phage type (only phage types shared by more than two isolates are indicated). Numbers on branches indicate the number of loci that vary between each MLVA profile. Grey shading indicates clonal complexes created based on maximum neighbour distance of changes at one locus and a minimum of five MLVA profiles per complex. 
TABLE 2

Comparison of the ten most common MLVA profiles among Salmonella Typhimurium monophasic isolates with clonal complex and phage type, England and Wales, 2010 $(n=139$ isolates $)$

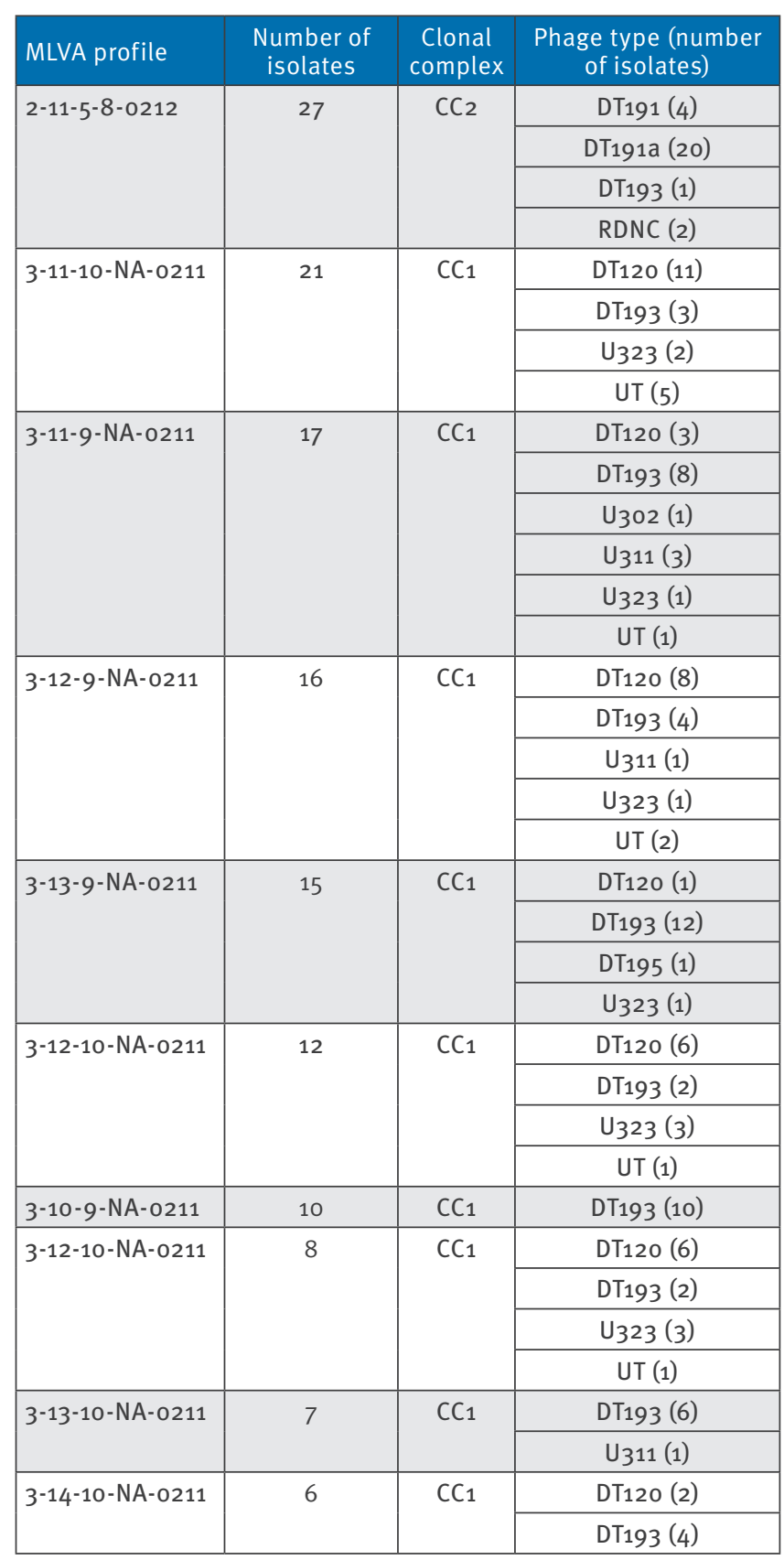

CC: clonal complex; DT: definitive phage type; MLVA: multiple locus variable-number tandem repeat analysis; NA: not amplified; RDNC: reacts but does not conform; UT: untypable. the island. However, a further $38 \%$ produced a single amplicon of $903 \mathrm{bp}$, suggesting mutation or deletion occurring either upstream of the inserted island or in the 5' end of the island itself (leading to a 'partial island'), or within the primer binding sites. In a random selection of six isolates producing only the 903bp band, five isolates supported amplification of the $1,128 \mathrm{bp}$ band when tested in a monoplex PCR with a slightly lowered primer annealing temperature (data not shown). This suggests that for at least some isolates, the absence of the $1,128 \mathrm{bp}$ band when testing using the multiplex PCR is due to mutation(s) within the primer binding site(s). In addition, the complete or 'partial island' was identified in $25 \mathrm{fljB}+/ \mathrm{hin}+$ DT193 isolates and nearly three quarters of monophasic isolates belonging DT120, UT, U323 and DT195. Given that all but one isolate harbouring the genomic island clustered together in $\mathrm{CC}_{1}$ regardless of antigenic structure and phage type, and assuming that all 17 open reading frames (ORFs) that make up the genomic island are present in these strains, these $f l j B+/ h i n+D T 193$ isolates may be progenitors of the fljB-/hin-DT193 isolates. In turn, these may have changed phage type by plasmid loss or acquisition, lysogenic conversion or alterations in lipopolysaccharide to account for the presence of the genomic island in genetically related monophasic isolates of other phage types. Trüpschuch et al. hypothesised that uptake of the island occurred in two or more steps [15]; if this is the case it seems unlikely that the genomic island would have been acquired through multiple independent genetic events.

Monophasic variants of serovar Typhimurium have already caused substantial outbreaks in several countries and continue to pose a public health risk $[10,11,13,26]$. Reliable detection of monophasic variants of serovar Typhimurium is important to ascertain the impact the emergence of these strains is having on the food chain and the number of human infections, and to monitor control efforts. Legislation of the EU has now been redrafted to include serovar 4,[5],12:i:in actions taken to detect and control Salmonella serovars of public health significance in laying hens (Commission Regulation (EU) No. 517/2011). In order to more accurately identify these isolates, the HPA SRU has been determining the full antigenic structure of all presumptive 0:4 isolates since the beginning of 2012 in addition to performing phage typing for identification of serovar Typhimurium and its variants. Isolates will be passaged through single strength Craigie's tubes, followed by double strength Craigie's tubes if a negative result is obtained after the first attempt at flagellar phase inversion. At present molecular methods will not be applied due to the large number of isolates received in the laboratory, but new molecular methods for identification of serovar Typhimurium and its variants will be assessed as they become available. 


\section{References}

1. European Food Safety Authority (EFSA) Panel on Biological Hazards (BIOHAZ). Scientific Opinion on monitoring and assessment of the public health risk of "Salmonella Typhimurium-like” strains. EFSA Journal. 2010;8(10):1826. http://www.efsa.europa.eu/en/scdocs/doc/1826.pdf

2. European Food Safety Authority. The community summary report on trends and sources of zoonoses, zoonotic agents, antimicrobial resistance and foodborne outbreaks in the European Union in 2006. EFSA Journal. 2007;130. Available from: http://www.efsa.europa.eu/en/scdocs/scdoc/13or.htm

3. Echeita MA, Herrera S, Usera MA. Atypical, fljB-negative Salmonella enterica subsp. enterica strain of serovar $4,5,12$ : i:appears to be a monophasic variant of serovar Typhimurium. Clin Microbiol. 2001;39(8):2981-3.

4. Dionisi AM, Graziani C, Lucarelli C, Filetici E, Villa L, Owczarek $S$ et al. Molecular characterization of multidrug-resistant strains of Salmonella enterica serotype Typhimurium and Monophasic variant (S. 4,[5],12:i:-) isolated from human infections in Italy. Foodborne Pathog Dis. 2009;6(6):711-7.

5. Soyer Y, Moreno SA, Davis MA, Maurer J, McDonough PL, Schoonmaker-Bopp DJ et al. Salmonella 4,5,12:i:-: an emerging Salmonella serotype that represents multiple distinct clones. J Clin Microbiol. 2009;47(11):3546-56.

6. Anderson ES, Ward LR, Saxe MJ, de Sa JD. Bacteriophagetyping designations of Salmonella typhimurium. J Hyg (Lond). 1977;78(2):297-300.

7. Hopkins KL, Kirchner M, Guerra B, Granier SA, Lucarelli C, Porrero MC et al. Multiresistant Salmonella enterica serovar 4,[5],12:i:- in Europe: a new pandemic strain? Euro Surveill. 2010;15(22):pii=19580. Available from: http://www. eurosurveillance.org/ViewArticle.aspx?Articleld =19580

8. Echeita MA, Aladueña A, Cruchaga S, Usera MA. Emergence and spread of an atypical Salmonella enterica subsp. enterica serotype 4,5,12:i:- strain in Spain. J Clin Microbiol. 1999;37(10):3425.

9. Hauser E, Tietze E, Helmuth R, Junker E, Blank K, Prager R et al. Pork contaminated with Salmonella enterica serovar $4,[5], 12: \mathrm{i}$ :, an emerging health risk for humans. Appl Environ Microbiol. 2010;76(14):4601-10.

10. Bone A, Noel H, Le Hello S, Pihier N, Danan C, Raguenaud ME et al. Nationwide outbreak of Salmonella enterica serotype 4,12:i:- infections in France, linked to dried pork sausage, March-May 2010. Euro Surveill. 2010;15 (24): pii=19592. Available from: http://www.eurosurveillance.org/ViewArticle. aspx?Articleld=19592

11. Mossong J, Marques P, Ragimbeau C, Huberty-Krau P, Losch $\mathrm{S}$, Meyer $\mathrm{G}$ et al. Outbreaks of monophasic Salmonella enterica serovar 4,[5],12:i:- in Luxembourg, 2006. Euro Surveill. 2007;12(6): pii=719. Available from: http://www. eurosurveillance.org/ViewArticle.aspx?Articleld=719

12. Harker KS, Lane C, de Pinna E, Adak GK. An outbreak of Salmonella Typhimurium DT191a associated with reptile feeder mice. Epidemiol Infect. 2011;139(8):1254-61.

13. Agasan A, Kornblum J, Williams G, Pratt CC, Fleckenstein $P$, Wong $M$ et al. Profile of Salmonella enterica subsp. enterica (subspecies I) serotype $4,5,12$ : i:- strains causing food-borne infections in New York City. J Clin Microbiol. 2002;40(6):1924-9.

14. Tavechio AT, Ghilardi AC, Fernandes SA. "Multiplex PCR" identification of the atypical and monophasic Salmonella enterica subsp. enterica serotype 1,4,[5],12:i:- in São Paulo State, Brazil: frequency and antibiotic resistance patterns. Rev Inst Med Trop Sao Paulo. 2004;46(2):115-7.

15. Trüpschuch S, Laverde Gomez JA, Ediberidze I, Flieger A, Rabsch W. Characterisation of multidrug-resistant Salmonella Typhimurium 4,[5],12:i:- DT193 strains carrying a novel genomic island adjacent to the thrW tRNA locus. Int J Med Microbiol. 2010;300(5):279-88.

16. Bale JA, de Pinna EM, Threlfall EJ, Ward LR. Kauffmann-White Scheme - 2007: Salmonella Identification - Serotypes and Antigenic Formulae. London: Health Protection Agency; 2007.

17. Amavisit $P$, Boonyawiwat $W$, Bangtrakulnont $A$. Characterization of Salmonella enterica serovar Typhimurium and monophasic Salmonella serovar 1,4,[5],12:i:- isolates in Thailand. J Clin Microbiol. 2005;43(6):2736-40.

18. Lindstedt BA, Vardund T, Aas L, Kapperud G. Multiple-locus variable-number tandem-repeats analysis of Salmonella enterica subsp. enterica serovar Typhimurium using PCR multiplexing and multicolor capillary electrophoresis. J Microbiol Methods. 2004;59(2):163-72.

19. Larsson JT, Torpdahl M, Petersen RF, Sorensen G, Lindstedt BA, Nielsen EM. Development of a new nomenclature for Salmonella Typhimurium multilocus variable number of tandem repeats analysis (MLVA). Euro Surveill. 2009;14(15):pii=19174.
Available from: http://www.eurosurveillance.org/ViewArticle. aspx?Articleld $=19174$

20. Echeita MA, Herrera S, Garaizar J, Usera MA. Multiplex PCR-based detection and identification of the most common Salmonella second-phase flagellar antigens. Res Microbiol. 2002;153(2):107-13.

21. Tennant SM, Diallo S, Levy H, Livio S, Sow SO, Tapia M et al. Identification by PCR of non-typhoidal Salmonella enterica serovars associated with invasive infections among febrile patients in Mali. PLoS Negl Trop Dis. 2010;4:e621.

22. Barco L, Lettini AA, Ramon E, Longo A, Saccardin C, Pozza MC et al. A rapid and sensitive method to identify and differentiate Salmonella enterica serotype Typhimurium and Salmonella enterica serotype 4,[5],12:i:- by combining traditional serotyping and multiplex polymerase chain reaction. Foodborne Pathog Dis. 2011;8(6):741-3.

23. Zamperini K, Soni V, Waltman D, Sanchez S, Theriault EC, Bray J et al. Molecular characterization reveals Salmonella enterica serovar $4,[5], 12$ :i:- from poultry is a variant Typhimurium serovar. Avian Dis. 2007;51(4):958-64.

24. Hopkins KL, Nair S, Kirchner M, Guerra B, Granier SA, Lucarelli $C$ et al. Genetic variation in emerging multidrug-resistant Salmonella enterica 4,[5],12:i:- from seven European countries. 2nd ASM Conference on Antimicrobial Resistance in Zoonotic Bacteria and Foodborne Pathogens in Animals, Humans and the Environment; 2010 Jun 8-11; Toronto, Canada.

25. Lucarelli C, Dionisi AM, Filetici E, Owczarek S, Luzzi I, Villa L. Nucleotide sequence of the chromosomal region conferring multidrug resistance (R-type ASSuT) in Salmonella Typhimurium and monophasic Salmonella Typhimurium strains. J Antimicrob Chemother. 2012;67(1):111-4.

26. Peters T, Hopkins KL, Lane C, Nair S, Wain J, de Pinna E. Emergence and characterization of Salmonella enterica serovar Typhimurium phage type DT191a. J Clin Microbiol. 2010;48(9):3375-7. 